\title{
Using of Scattering Bond Graph Methodology for a Physical Characteristics Analysis of "D-CRLH" Transmission Line
}

\author{
Hichem Taghouti ${ }^{\dagger}$, Sabri Jmal* and Abdelkader Mami*
}

\begin{abstract}
In this paper, we propose to analyze the physical characteristics of a planar dualcomposite right-left handed transmission line by a common application of Bond Graph approach and Scattering formalism (Methodology S.BG). The technique, we propose consists, on the one hand, of modeling of a dual composite right-left metamaterial transmission line (D-CRLH-TL) by Bond Graph approach, and, it consists of extracting the equivalent circuit of this studied structure. On the other hand, it consists to exploiting the scattering parameters (Scattering matrix) of the DCRLH-TL using the methodology which we previously developed since 2009. Finally, the validation of the proposed and used technique is carried out by comparisons between the simulations results with ADS and Maple (or MatLab).
\end{abstract}

Keywords: Bond graph modeling, Metamaterials transmission line, Composite right/left handed, Scattering parameters

\section{Introduction}

This reduction of size in electronic circuits (antenna and microwave circuits) is becoming increasingly important in the production of small-size millimeter-wave systems [1]. This led us, nowadays, to increase the use of metamaterials technology for the production and development of transmission lines (TL) [2]. To obtain the required compact sizes and a multi-band functionality, the producers of electronic circuits operating at high frequencies, often, prefer to use composite right/left handed (CRLH) transmission lines in particular the dual-CRLH transmission lines [3], principally, to the design of dual-band impedance inverters, as well as wideband band pass filters. Unfortunately, despite the advantage of the metamaterials the use of this technology [4] presents a major problem in the analysis of physical characteristics (such as the reflection and transmission coefficients, dispersion relation and frequency response) and electrical modeling of these types of electronic circuits and antennas. The major problem revealed when changing parameters, structure, equivalent circuit of metamaterial transmission line that we want to make, it forces us to redo the work (studies) from the start without being sure of the efficiency of the found results. For this reason, this paper is focused on the study of new analysis and modeling technique for D-CRLH transmission lines based on the jointly use of the scattering formalism and the bond graph approach often called "Scattering Bond Graph Methodology" [5]. The proposed method is fast, effective

$\dagger \quad$ Corresponding Author: Dept. of Electrical Engineering, El Manar II University, ENIT, Tunisia. (hichem.taghouti@live.fr)

* Dept. of Electrical Engineering and Physics, El Manar II University, Faculty of Sciences, Tunisia. (sabri.jmal@gmail.com, Abdelkader. mami@fst.rnu.tn)

Received: November 21, 2015; Accepted: January 3, 2016 and does not need to repeat the study of the system in case of conflict.

The bond graph approach [6] is a multidisciplinary analysis and modeling tool of physical systems (automatic, electric, acoustic, mechanical and thermodynamic ...), it is within the reach of all non-specialists. It constitute a major asset in its use, jointly with the scattering formalism [7], (in the study of systems operating at high frequencies: microwave circuits, antenna patch, open split ring resonators OSRRs and complementary split ring resonators CSRRs) [8].

Throughout this paper, we present our own analysis technique of physical characteristics of a dual composite right/left handed transmission line using the scattering bond graph methodology.

First, we will present the procedure of modeling technique of unit cell CRLH transmission line by bond graph approach. Second, we will apply this technique to a dual CRLH-TL. Then, we will extract directly the equivalent circuit of the proposed cell from its bond graph model. Finally, we will analyze the characteristics of the studied structure by exploiting its scattering parameters $S_{11}$, $\mathrm{S}_{12}, \mathrm{~S}_{21}, \mathrm{~S}_{22}$ based on the analytical exploitation procedure of the scattering parameters developed in [9].

To demonstrate and validate our technique, a comparative study, by simulation of the founded results, is proposed. In this work we will use the ADS and Maple (or MATLAB) for simulations [10].

\section{Bond Graph Modeling of CRLH Transmission Line}

\subsection{Metamaterials transmission lines and CRLH}

Unfortunately, there is no specific definition of the term 
"metamaterials". But, referring to the works of Caloz [11], Solymar and Shamonina [12], metamaterials are defined as artificial effectively homogeneous electromagnetic structures with unusual properties not readily available in nature. Therefore, they consider "metamaterials" like those artificial structures in which the unit cell size is much smaller than the guided wavelength of the effective artificial medium [13]. This technology can be implemented and used in various microwave applications and in different planar or multi-layer technologies; indeed, a relation between the effective parameters of metamaterials with those of transmission line is required. For this reason, metamaterials can be analyzed by means of transmission line theory [14].

Although all the artificial transmission lines analyzed so far present either left or right handed wave propagation, it is also possible to design artificial structures with both left and right handed wave propagation depending on the frequency region. These lines are called composite right / left-handed transmission lines (CRLH-TL) [15, 26].

\subsection{Bond graph modeling of CRLH-TL}

In recent article [5, 21], we studied the characteristics of a transmission line section by the bond graph approach. It behaves like a combination of a series impedance $Z$ in conjunction (shunt) with a shunt admittance $Y$ (and vice versa) [16], like the Fig. 1 and Fig. 2 indicate it.

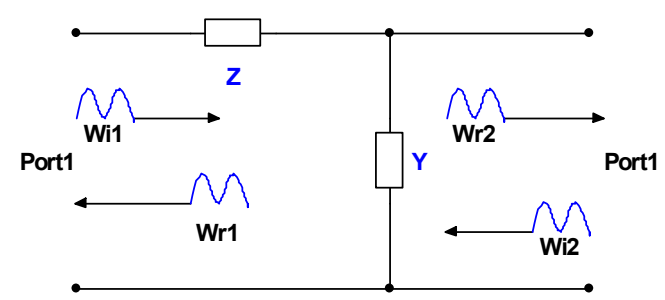

Fig. 1. The proposed model of TL in its ZY structure with incident and reflected waves

The second structure is given by the Fig. 2 .

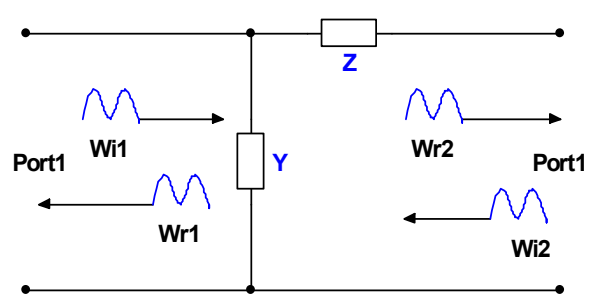

Fig. 2. The proposed model of TL in its YZ structure with incident and reflected waves

Where: $\mathrm{w}_{\mathrm{i} 1}$ and $\mathrm{w}_{\mathrm{i} 2}$ are the incident wave at port 1 and port $2, \mathrm{w}_{\mathrm{r} 1}$ and $\mathrm{w}_{\mathrm{r} 2}$ the reflected wave at port 1 and port 2 .

The non-causal bond graph model of the transmission line is given bellow. The first type of the bond graph model is often noted BGMZY and it is given by the following figure:

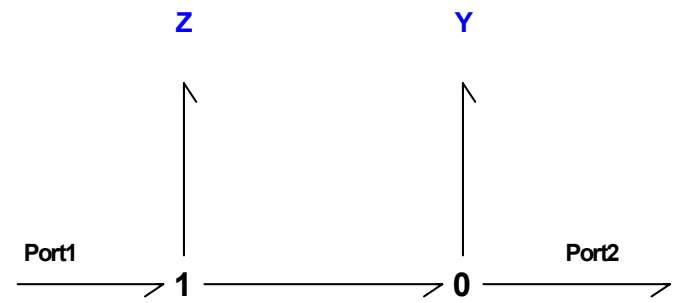

Fig. 3. A non-causal Bond Graph model of TL section (Often noted: BGMZY)

The second type of bond graph model, often noted BGMYZ, is given by the following figure:

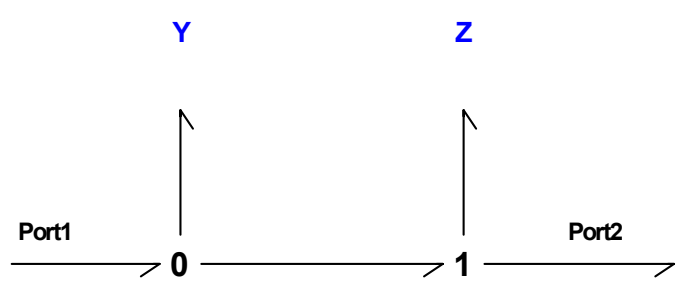

Fig. 4. A non-causal Bond Graph Model of TL section (Often noted: BGMYZ)

Series impedance $\mathrm{Z}$ and Shunt admittance $\mathrm{Y}$ can take many forms.

- If series $\mathrm{Z}$ and shunt $\mathrm{Y}$ have, respectively, the behaviors of capacitance (C) and inductance (L), the transmission line will be a homogeneous left handed transmission line (LH-TL) [17].

- If the series $Z$ and shunt $Y$ have, respectively, the behaviors of inductance (L) and capacitance (C), the transmission line will be a homogeneous right handed transmission line (RH-TL) [17].

While, the composite right left handed transmission line (CRLH-TL) [18] is given by the combination of RHtransmission line and LH-transmission line.

When the CRLH-TL is used for an antenna, there are backward wave in the lower frequencies and forward wave in the higher frequencies.

The model of CRLH-TL in its ZY structure with the incident and reflected waves at the entry and the exit ports is given by the following figure.

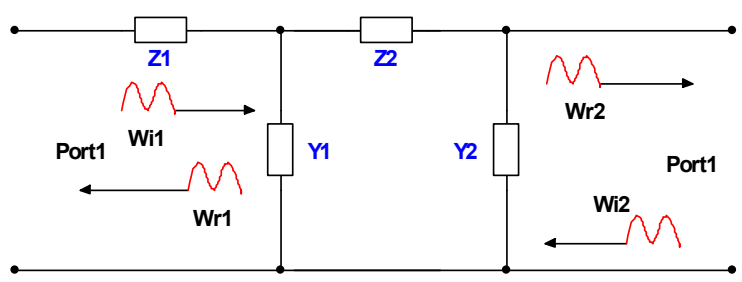

Fig. 5. ZY structure of the CRLH-TL with all waves 
Note that the CRLH-TL can take other structure often called $\mathrm{YZ}$ structure.

The bond graph model of the previously structure is given bellow.

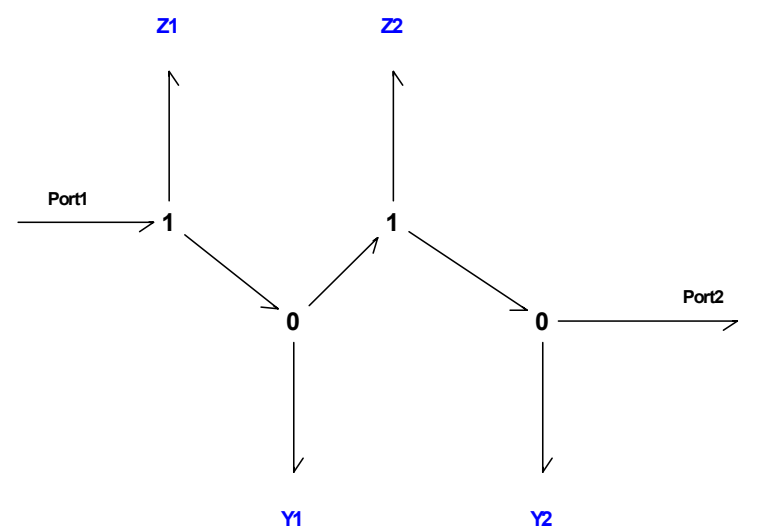

Fig. 6. A non-causal bond graph model of CRLH-TL structure

where:

$$
\begin{aligned}
& Z_{1}=Z_{2}=\frac{1}{j C \omega}+j L \omega \\
& Y_{1}=Y_{2}=\frac{1}{j C \omega+\frac{1}{j L \omega}}
\end{aligned}
$$

$\mathrm{C}$ and $\mathrm{L}$ are respectively the capacitance and the inductance behaviors of the CRLH-TL.

\subsection{Scattering bond graph analysis}

The analysis of the physical characteristics of any structure (antenna, microwave circuits...) by scattering bond graph approach constitute to extract, directly, the scattering parameters from the reduced and causal BG model of this structure by respecting the rules developed in [19]. From those scattering parameters $\left(\mathrm{S}_{11}, \mathrm{~S}_{12}, \mathrm{~S}_{21}, \mathrm{~S}_{22}\right)$, we can perform other analyzes such us: dispersion relation, frequency response. The new analysis technique consists, first, to affect the reduced causality to non-causal Bond Graph model. Then, to put the model in the form of a bond graph cell intercalated between the input port 1 and the output port 2. In the case where the system is complex, the decomposition in the form of a cascade bond graph cells takes place. The extracting of the scattering matrix is performed by an analytical method that we developed in [19]. The causal and global bond graph model of CRLHTL is given bellow.

Among the important properties of the bond graph approach is that it gives us the right to simplify the model without changing its physical behavior, in particular the spread of incident and reflected waves through the system [20]. Hence the new simplified model the following figure:

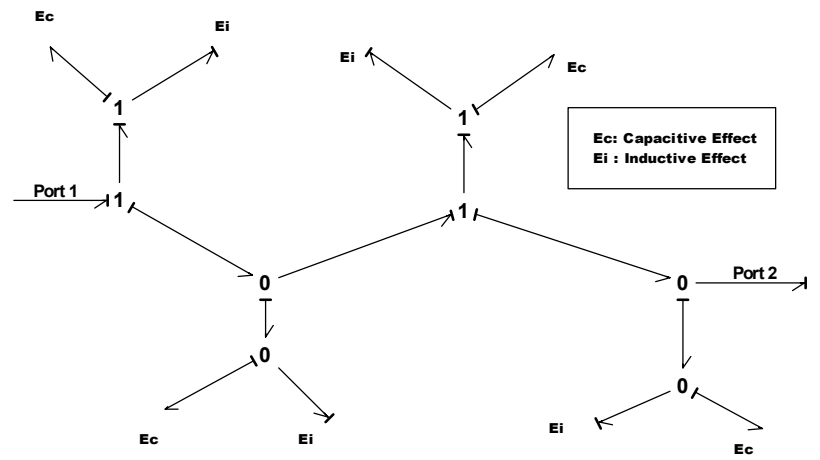

Fig. 7. Global and causal bond graph model of CRLH-TL structure

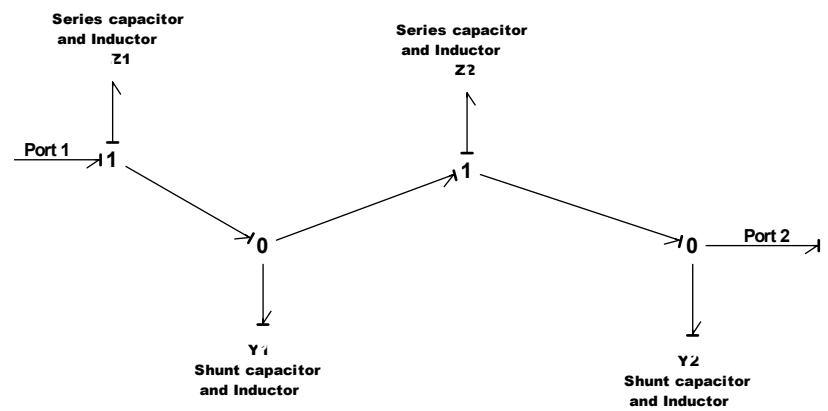

Fig. 8. The simplified and causal bond graph model of CRLH-TL structure

The decomposition of the above model in the form of sub-model $\mathrm{BGM}_{\mathrm{ZY}}$, leads us to the following figure:

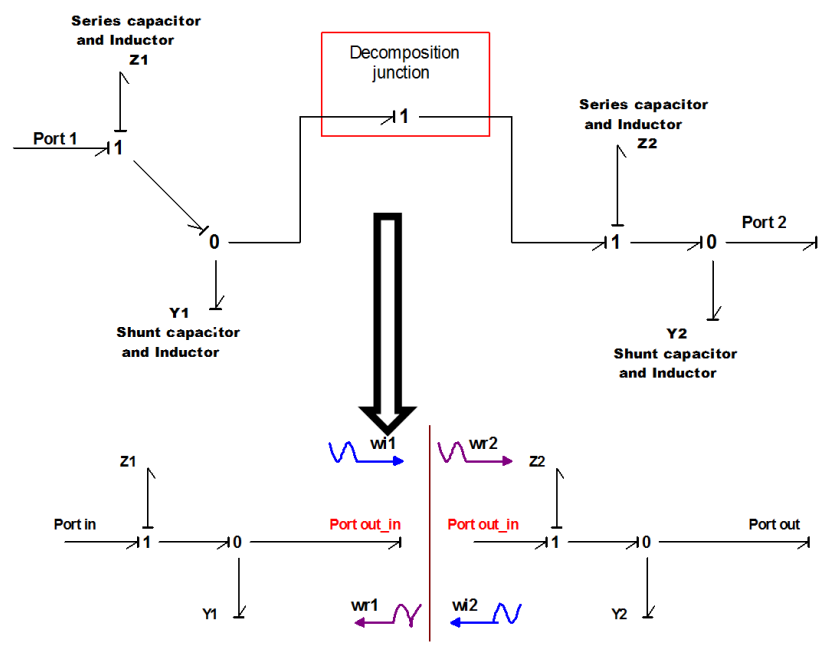

Fig. 9. The tow Cells (1-0 junction) of $\mathrm{BGM}_{\mathrm{ZY}}$

From each cell $\left(\mathrm{BGM}_{\mathrm{ZY}}\right)$, we can extract, directly, the wave matrix $\mathrm{W}_{z y}[21]$.

$$
W_{z y}=\left[\begin{array}{cc}
\frac{z y+z+y+2}{2} & \frac{z y-z+y}{2} \\
\frac{z y+z-y}{2} & \frac{z y-z-y+2}{2}
\end{array}\right]
$$


And the scattering matrix $S$ can be deduced directly from the wave matrix $W .[21]$

$$
S_{z y}=\frac{1}{z y+z+y+2}\left[\begin{array}{cc}
z y+z-y & 2 \\
2 & -z y+z-y
\end{array}\right]
$$

The scattering matrix of two cascaded systems is not the product of matrices of each system, that's why we often use the product of the wave matrices of each system and then we perform the conversion to $\mathrm{S}$ matrix by methods we develop in [22]. The overall wave matrix of two systems (two cells -1-0- or -0-1- ) cascade is:

$$
\begin{aligned}
& W_{z y}^{T}=W_{z y}^{1} \times W_{z y}^{2}=\left[\begin{array}{ll}
W_{11} & W_{12} \\
W_{21} & W_{22}
\end{array}\right] \\
& W_{z y}^{T} \stackrel{\begin{array}{l}
\text { by conversion with } \\
\text { analytical method }
\end{array}}{\longrightarrow} S_{z y}^{T} \\
& \text { Or: } \quad W_{y z}^{T} \stackrel{\begin{array}{l}
\text { by conversion with } \\
\text { analytical method }
\end{array}}{\longrightarrow} S_{y z}^{T} \\
& S_{z y}^{T}=\left[\begin{array}{ll}
S_{11} & S_{12} \\
S_{21} & S_{22}
\end{array}\right] \\
& \left\{\begin{array}{l}
S_{11}=\frac{W_{21}}{W_{11}} \\
S_{12}=\frac{W_{11} W_{22}-W_{12} W_{21}}{W_{11}} \\
S_{21}=\frac{1}{W_{11}} \\
S_{22}=-\frac{W_{12}}{W_{22}}
\end{array}\right.
\end{aligned}
$$

\section{Physical Characteristics Study of D-CRLH-TL by Bond Graph Approach}

The physical characteristics analysis of the D-CRLH unit by bond graph approach, consists of determine the frequency response and the dispersion relation of this structure. This determination is performed after completing the bond graph model of the structure and then to extract the scattering matrix by the analytical methods developed in [5].

The famous goal of using the bond graph approach, which facilitates the determination of equivalent circuit of the D-CRLH structure, which will lead in turn to other important analyzes.

\subsection{Scattering bond graph analysis: Study and results}

We take the example of the structure proposed by Liu, Q.-X. Chu, and J.-Q. Huang in their paper [23], where they considered that the D-CRLH transmission line consists

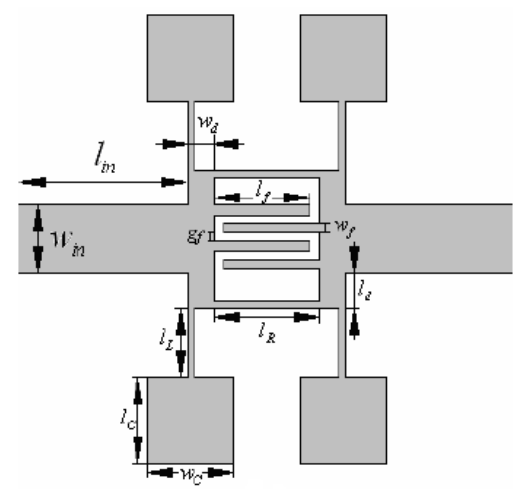

Fig. 10. A unit cell of the D-CRLH transmission line structure $[23,28]$

of one interdigital capacitor paralleled by two high impedance stubs, and four series branches of the plane capacitor and stub [23].

The analysis $[27,10,28]$ of the above structure, will lead us to build its global bond graph model as shown in the following figure.

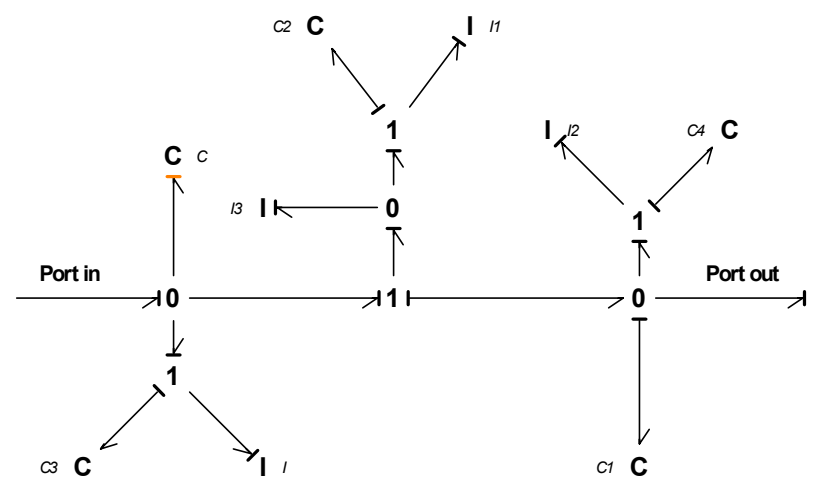

Fig. 11. Causal Bond Graph model of the D-CRLH transmission line structure

Where: $\mathrm{C}$ and I are, respectively, the capacitive and the inductive effect through the D-CRLH TL.

- Interpretation: The bond graph approach graph model the structure as a parallel branch (junction 0) of a parasitic capacitor $C_{p}$ in parallel with an inductance and capacitance $\mathrm{L}_{\mathrm{s}}$ and $\mathrm{C}_{\mathrm{s}}$ series. This branch, in series with another series branch (junction 1) bringing together in parallel $\mathrm{L}_{\mathrm{sb}}$ inductance (which models the stub) with the inductance $\mathrm{L}_{\mathrm{p}}$ (inductive parasitic effects) and the $\mathrm{C}^{*} \mathrm{~s}$ series capacitance (series capacitive effects) [10]. As the structure is symmetric, we add the same input branch (parallel branch) to the output. Hence the bond graph model with all the resonant elements of the D-CRLH TL structure. [24]

Based on the properties of bond graph approach, the above model becomes a reduced and simplified model $\left(\mathrm{BGM}_{\mathrm{YZ}}\right)$ as shown in the following figure. 


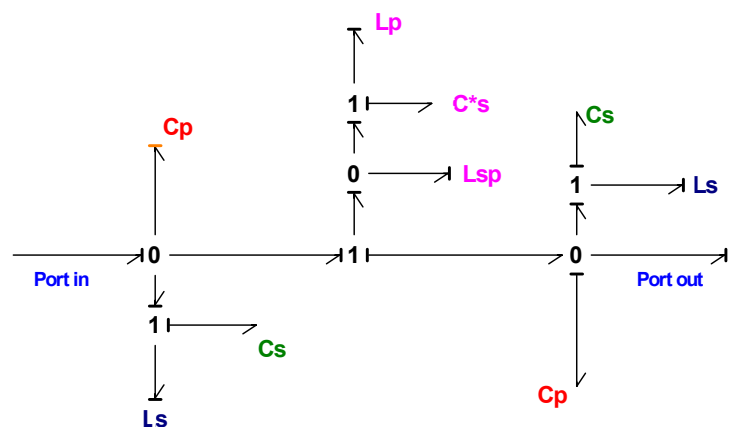

Fig. 12. Bond Graph model of the D-CRLH with all resonant elements

$$
\begin{gathered}
Y_{1}=\frac{1}{j C_{p} \omega} \\
Y_{2}=j L_{s b} \omega \\
Z_{1}=j L_{p} \omega+\frac{1}{j C_{s} \omega} \\
Z_{2}=j L_{p} \omega+\frac{1}{j C_{s}^{*} \omega}
\end{gathered}
$$

The normalization [7] of the resonant elements according to the internal impedance $Z_{0}$ gives us the reduced values of the bond graph approach such as the following equations:

$$
\begin{gathered}
y=\tau_{c p} s+\frac{1}{\tau_{L s} s+\tau_{C s} s} \\
z=\frac{1}{\tau_{L s b} s}+\frac{1}{\tau_{L p} s+\tau_{C s} s}
\end{gathered}
$$

$s$ : Laplace operator,

$\tau_{X}=Z_{0} \cdot Z_{X}$ : The normalized element constant.

From the Fig. 13, we can extract the wave matrix $W_{y z}$ of the structure, which can give us the Scattering parameters $\left(\mathrm{S}_{11}, \mathrm{~S}_{12}, \mathrm{~S}_{21}\right.$ and $\left.\mathrm{S}_{22}\right)$.

$$
\begin{gathered}
W_{y z}=\left[\begin{array}{ll}
w_{11} & w_{12} \\
w_{21} & w_{22}
\end{array}\right] \\
w_{11}=\frac{y^{2}+2 z y+2(z+y)+1}{2 z} \\
w_{21}=\frac{y^{2}+2 z y-1}{2 z} \\
w_{21}=\frac{-y^{2}-2 z y+1}{2 z} \\
w_{22}=\frac{-y^{2}-2 z y+2(z+y)+1}{2 z}
\end{gathered}
$$

The scattering parameters, by referring to equations 8 and 9 , are given bellow.

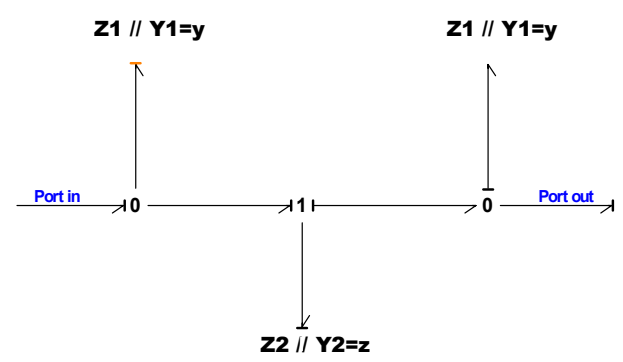

Fig. 13. Reduced and simplified Bond Graph model of the D-CRLH

$$
\begin{aligned}
& S_{11}=\frac{-y^{2}-2 z y+1}{y^{2}+2 z y+2(z+y)+1} \\
& S_{12}=\frac{2 z}{y^{2}+2 z y+2(z+y)+1} \\
& S_{21}=\frac{2 z}{y^{2}+2 z y+2(z+y)+1} \\
& S_{22}=\frac{-y^{2}-2 z y+1}{y^{2}+2 z y+2(z+y)+1}
\end{aligned}
$$

\subsection{Experiment study and comparisons}

The experimental results of D-CRLH TL, performed by Liu, Q.-X. Chu, and J.-Q. Huang in their paper [23], are given below, where they used the HFSS and a mathematical approximations to realize and simulate the equivalent circuit of the proposed system.

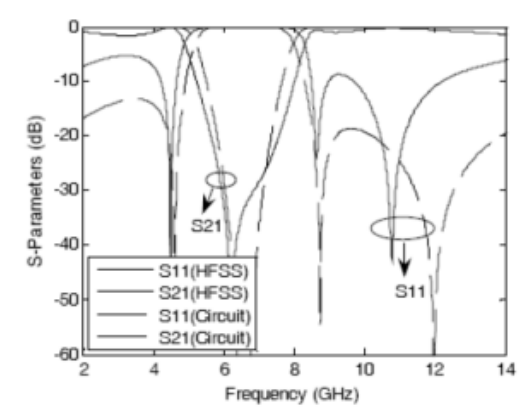

Fig. 14. $S_{11}$ and $S_{21}$ parameters of the proposed D- CRLH TL [23]

Our discussion is to refuse the mathematical approximations used to produce such a circuit equivalent since they cannot provide precise and even inaccurate results. Hence the object of the use of the bond graph approach that provides accurate equivalent circuits and leads to accurate simulations and the phenomenon is reversible [28, 24]. We note that Liu, Q. et al. in their paper [23], used HFSS and the approximations of the equivalent circuit to find the results above. Our research team uses the scattering bond graph method to find the same results that we are looking for, we can evaluate and simulate the 


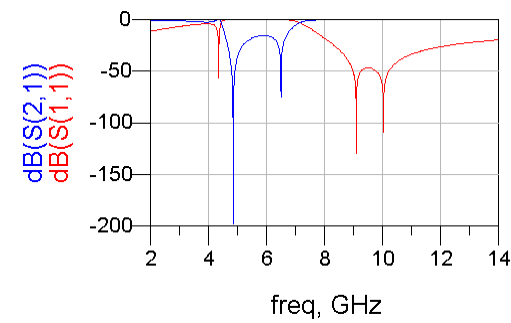

Fig. 15. Simulation results of $S_{11}$ and $S_{21}$ parameters by BGM

scattering parameters by our own method, and we can also extract the equivalent circuit any model simple or complex $[9-10]$.

\subsection{Equivalent circuit exploitation of D-CRLH TL}

The exploitation of the equivalent circuit of the DCRLH TL, by bond graph approach, is to use the reverse properties of this approach.

From Fig. 11, we can give directly the equivalent circuit of the D-CRLH TL.
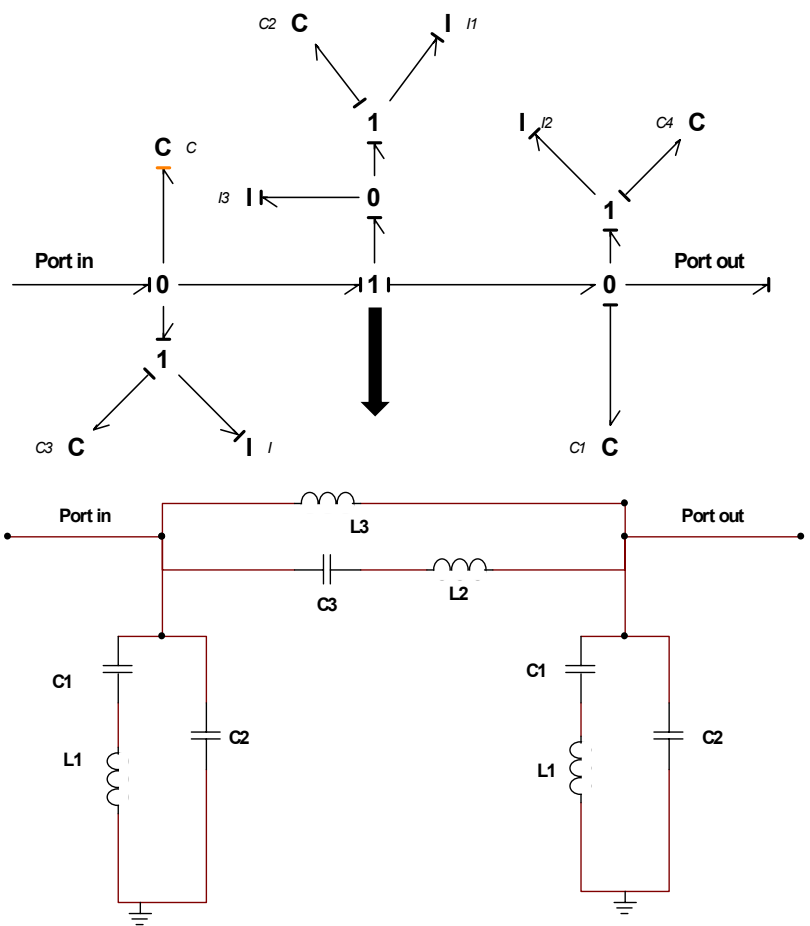

Fig. 16. From Bond Graph to equivalent circuit of DCRLH TL

The reverse properties of Bond Graph approach [27, 28], consists of transform the capacitive and inductive effect to lumped elements [25] by taking into account the equations developed in our recent article [24].

$$
L(n H)=2 \cdot 10^{-4} \ell\left[\ln \left(\frac{\ell}{W+t}\right)+1,193+\frac{W+t}{3 \ell}\right] \cdot K_{g}
$$

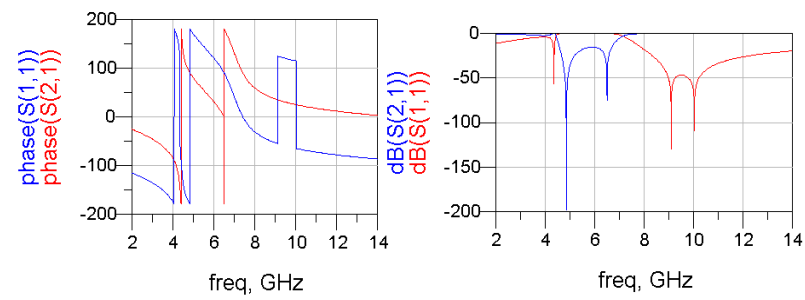

Fig. 17. Simulation results of equivalent circuit by BGM

$$
\begin{gathered}
R_{s}(\Omega)=\frac{K \cdot R_{s h} \ell}{2 \cdot(W+t)} \\
C_{1}(p F)=16,67 \cdot 10^{-4} \ell \sqrt{\frac{\varepsilon_{r e}}{Z_{0}}}
\end{gathered}
$$

$K \quad$ : a correction factor.

$Z_{0}:$ Characteristic impedance.

$\varepsilon_{r e}:$ Effective dielectric constant.

$$
\begin{gathered}
K_{g}=0,57-0,145 \cdot \ln \frac{W}{h}, \frac{W}{h}>0,05 \\
K=1,4+0,217 \cdot \ln \left(\frac{W}{5 t}\right)
\end{gathered}
$$

$5<\frac{W}{t}<100$. For a ribbon

$$
K=1+0,333\left(1+\frac{S}{W}\right) . \text { For a spiral }
$$

\section{Conclusion}

A scattering Bond Graph methodology is proposed. This methodology is simple, flexible and fast which facilitates the study of many types of transmission line. It can give us an equivalent circuit which can be fabricated by using lumped elements and with minor error. This method is very easy for a non-specialist staff how find, always many problems in analysis, design and electrical modeling study. Agreements between equivalent circuit simulated and scattering bond graph parameters responses are demonstrated.

\section{Acknowledgements}

We note that this project is supported by ENIT (LR11ES20) and laboratory of high frequency and electronic circuits systems $(05 / \mathrm{UR} / 11-10)$.

\section{References}

[1] Y. Cao, G. Wang, and Q. J. Zhang, “A new training approach for parametric modeling of microwave 
passive components using combined neural networks and transfer functions", Microwave Theory and Techniques, IEEE Transactions on, Vol. 57, No. 11, pp. 2727-2742, 2009

[2] C. Caloz, and T. Itoh, "Electromagnetic Metamaterials: Transmission Line Theory and Microwave Applications", Wiley-IEEE Press, New York, ISBN: 978-0-471-66985-2, pp. 376, December 2005.

[3] S. Simon, R. M., G. Bartolucci, “Composite Right / Left Handed (CRLH) Based Devices for Microwave Applications", ISBN:978-953-307-031-5, Published: March 1, under CC BY-NC-SA 3.0 license, 2010.

[4] J. -Q. Huang, Q. -X. Chu, and C. -Y. Liu, "Compact UWB filter based on surface-coupled structure with dual notched bands", Progress In Electromagnetics Research, Vol. 106, pp. 311-319, 2010.

[5] H.Taghouti, and A.Mami, "Discussion around the Scattering Matrix Realization of a Microwave Filter using the Bond Graph Approach and Scattering Formalism". American Journal of Applied Sciences, Vol. 9, Issue.4, 2012.

[6] W. Borutzky, "Bond Graph Modelling of Engineering Systems”, pp. 4-10. Springer, 2011.

[7] H. Taghouti, A. Mami, and S.Jmal, "Nouvelle Technique de Modélisation et Simulation par Bond Graph: Applications aux Circuits Hauts Fréquences et Antennes Patch". Éditions universitaires européennes, 2014.

[8] M. D.Sindreu, F. Aznar, A. Vélez, J. Bonache, F. Martín, "Analysis and applications of OSRR - and OCSRR-loaded transmission lines: A new path for the design of compact transmission line metamaterials", Vo. 4, Issues 2-3, August September, pp. 139-148, Metamaterials-2009, Congress in London, 2010.

[9] S. Jmal, H. Taghouti, , and A. Mami, "Modeling and simulation of a patch antenna from its Bond Graph model", International Conference on Control, Decision and Information Technologies (CoDIT), pp. 609-614. IEEE, 2014.

[10] S. Jmal, H. Taghouti, and A. Mami, "A new modeling and simulation methodology of a patch antenna by Bond Graph approach", International Conference on Electrical Engineering and Software Applications (ICEESA), pp. 1-6, IEEE, 2013.

[11] C. Caloz, T. Itoh, "Novel microwave devices and structures based on the transmission line approach of metamaterials", IEEE-MTT International Microwave Symposium, vol. 1, pp. 195-198, Philadelphia, PA, June (2003).

[12] L. Solymar and E. Shamonina, "Waves in Metamaterials", ISBN: 9780199215331, Oxford University press USA, 15 March 2009.
[13] F. Martín, F. Falcone, J. Bonache, R. Marqués, M. Sorolla, "Split ring resonator based left handed coplanar waveguide", Appl. Phys. Lett., Vol. 83, pp. 4652-4654, 2003.

[14] D.R. Smith, W.J. Padilla, D.C. Vier, S.C. NematNasser, S. Schultz, "Composite medium with simultaneously negative permeability and permittivity". Phys. Rev. Lett., Vol. 84, pp. 4184-4187,2000.

[15] M. Durán-Sindreu, F. Aznar, A. Vélez, J. Bonache, F. Martín, "New composite right/left handed transmission lines based on electrically small open resonators", IEEE-MTT-S International Microwave Symposium, Boston, USA, pp. 45-48, June 2009.

[16] H.Taghouti, and A. Mami, "Modeling Method of a Low-Pass Filter Based on Microstrip T-Lines with Cut-Off Frequency $10 \mathrm{GHz}$ by the Extraction of its Wave-Scattering Parameters from its Causal Bond Graph Model". Am. J. Eng. Applied Sci., Vol. 3, pp. 631-642, 2010.

[17] C. Caloz, and T. Itoh, "Transmission line approach of left-handed (LH) materials and microstrip implementation of an artificial LH transmission line", IEEE Transactions on Antennas and Propagation includes theoretical and experimental advances in antennas. Vol. 52, Issue: 5, pp. 1159-1166, DOI: 10. 1109/TAP. 2004.827249,2004.

[18] F. P. Casares-Miranda, E. Marquez-Segura, P. Otero, and C. Camacho Penalosa, "Composite right/lefthanded transmission line with wire bonded interdigital capacitor" IEEE Microwave Wireless Compon. Lett., Vol. 16, No. 11, Nov. 2006.

[19] S. Khmailia, H. Taghouti, R. Mehouachi and A. Mami, "Application of the reduced bond graph approach to determine the scattering parameters of a matching network of a planar inverted f antenna", International Journal of Advances in Engineering \& Technology, Vol. 3, Issue 2, pp. 77-89. May 2012.

[20] J. G. Proakis, M.Salehi, N. Zhou, and X. Li, "Communication systems engineering", Vol. 2. Englewood Cliffs: Prentice-hall, 1994.

[21] H. Taghouti and A. Mami, "New Extraction Method of the Scattering Parameters of a Physical System Starting From.

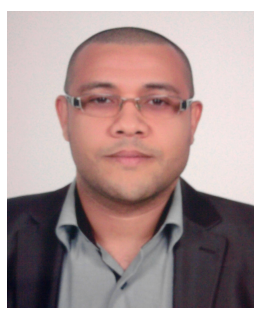

Hichem Taghouti is with High Institute of Environmental Sciences and Technologies (HIEST). His main research areas are the Bond Graph modelling and its new applications in high frequencies domain. Since 2011, Dr. Hichem TAGHOUTI was been an AssistantProfessor of Electrical Engineering. 


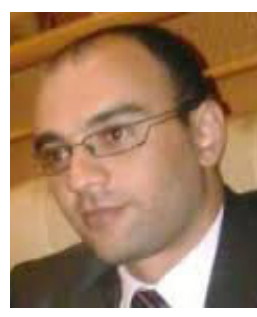

Sabri Jmal is a $\mathrm{PhD}$ student at University of Tunis El Manar, FST, Tunisia and Researcher AT National Engineering School of Tunis. Since 2014, he is teaching as an Assistant of Electronic and computer science in high institute of environment sciences and technologies of Borj Cedria Tunis

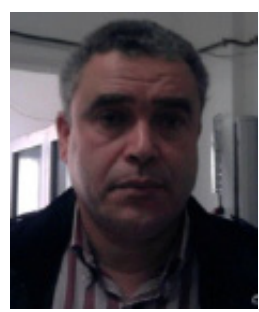

Abdelkader Mami is with University of Tunis El Manar, National Engineering School of Tunis. He is a Professor in Faculty of Sciences of Tunis (FST). He was received his HDR from the University of Lille. 